\title{
Professor Gayet †
}

Am 23. Juli 1904 wurde einer der bekanntesten Ophthalmologen der Gegenwart und ältesten Vertreter der französiscben Opbthalmologie, Professor A. Gayet zu Lyon, aus voller Arbeit dureh einen Wagenunfall plötzlich hínweggerafft.

Charles Jules Alphonse Gayet wurde am 19. Mai 1833 zu Saint Genis-Laval (Rhone) geboren. Er studierte Medizin in Lyo,, wurde daselbst 1854 Interne des hôpitaux, promovierte 1858 mit der These: Etude experi-mentale sur la ligature des artères und wurde 1861 zum Chirurgien major ernannt. 1865 erfolgte seine Berufung an Stelle von Chauveau zum Professor der Anatomie uod Physiologie an die medízinische Fakultät zu Lyon, 1870 nahm er als Chefarzt des zweiten Feldlazaretts der Loire - Armee am Feldzug teil, 1873 ward er zum Professor der Pathologie extern $\beta$ zu Lyon ernannt.

Während dieser chirurgischen und pathologisch-anatomischen Tätigkeit interessierte ihn sehon vorzugsweise die Ophthalmologie, er lernte sich selbst den Gebrauch des Augenspiegels und die Technik der Augen-operationen. Als dann 1877 der erste Lehrstuhl für Ophthalmologie in Frankreich zu Lyon gegründet wurde, wurde Gayet an diese Stelle bernfen, in der er bis zu seinem ïode eine reiche wissenschaftlicbe Lehrtätigkeit und augenärztliche Praxis ausgeübt hat. Gross ist die Zahl und Bedeutung Gayets wissenschaftlicher Arbeiten. Als exakter Forscher der normalen und pathologischen Histologie des Auges bearbeitete er fur das T > ictionnaire encyclopédique die Histologie der Cornea (1877) und der Linse (1879) und vollendete diese Studien über die Linse durch die Arbeit über die Histologie der Kapselkatarakt. 1876 erschien eine Arbeit über die Anatomie der Sklera, 1879 über die traumatischen Iris-cysten und 1885 in den Archives d'Ophthalmologie mit A. Masson zusammen die bedeutende Arbeit über die Ergebnisse seiner Studien der „Atrophie du globe oculaire”. Besondere Verdienste hat er sich erworben durch die Einführung der Mikrophotographie in die Ophthalmologie, deren Bedeutung er in den Arbeiten „La microphotographie dans les laboratoires” (1881) und in der „Iconographie photographique appliquée à l'ophthalmologique" (1887) dartat.

Unter seinen klinisehen Publikationen sind besonders hervorzuheben die erste Beobachtung eines Falles von akuter Ophthalmoplegie mit anatomi-schem Befund in der Arbeit ,Affection encéphalique localisée aux étages antérieures des pédoncules cér $1 / 8$ braux" (1875), die 1883 erschienene Arbeit über Retinitis punctata congenitalis mit Hemeralopie, die Studien über die sympathische Ophthalmic, in denen er sich der Theorie Deutschmanns anschliesst, und die beiden Veröffentlichungen über symmetrische Tumoren: „Sur les tumeurs symétriques des deux orbites” (1886) und „Un cas de tumeurs symétriques des globes oculaires” (1888), in denen er die rein lymphomatöse Natur dieser Tumoren feststellen konnte.

Immer auf Fortschritt bedacht, wendete er sich darauf dem Studium der Bakteriologie des Auges zu, veröffentlichte 1887 experimentelle Unter-suchungen über die Antisepsis und Asepsis des Auges und eine Beobachtung von infektiöser Panophthalmie.

Als gewandter und erfahrener Operateur hat er eine grosse Anzahl Operationsmethoden angegeben, iür Trichiasis und Entropium, für Ptosis,

Offene Korrespondenz. 
395

Pterygium und Staphyloma adhaerens, für das er die sogen. temporäre Um-kehrung der Hornhaut anwendete. Besonders interessierte ibn das Studium und die Operation der Katarakt. Er empfahl und übte die ãquatoriale Kapselöffnung zugleicb mit dem Schnitt, zwiscben Punktion und Gegen-punktion, und zog dem Eingeben mit dem Davielscben Löffel die Aus-spülung der vorderen Kammer nach Mac Kowen vor. Er veröffentlichte Untersuchungen über die Verbreitung der Katarakt in der Gegend von Lyon (1882), über die Wirkung der ultravioletten Strahlen bei Kataraktoperierten (1884) und über die Sehscbärfe naeh der Extraktion (1891). Von seinen grösseren didaktischen Arbeiten sind besonders die Ab-handlungen im Dictionnaire encyclopédique zu nennen, über die Cornea (1877), die Linse (1879), über Photophobie (1882), Hemeralopie (1888) und über Glaukom (1883), vor allem aber sein 1893 erschiene $\pi$ es Werk „Nouveaux elements d'Ophthalmologi à 1'usage des médecins pratiques”.

Zahlreiche Anerkennungen und Auszeichnungen wurden ihm für seine Verdienste um die ophthalmologische Wissenschaft zu teil. 1872 erbielt er für seine Verdienste im Pelde die Ehrenlegion, 1884 trat er in die Redaktion der Archives d'Ophthalmologie ein. Die französische Gesellschaft der medizinischen Wissenschaften ernannte ihn 1873 zum Präsidenten, die französische Gesellschaft für Chirurgie und die AcadémiedeMédecinezumkorrespondierenden Mitglied. 1900 war er zum Vizepräsidenten der ophthalmologischen Ab-teilung des internationalen Kongresses zu Paris, und 1902 zum Präsidenten derselben Abteilung des Kongresses zu Kairo ernannt worden; von 1900 bis 1902 war er President der französischen Gesellschaft für Chirurgie.

Gleich geliebt von seinen Schülern als Gelehrter, als Lehrer und als Mensch, hoch $3 / 8$ eachtet in der ganzen medizinischen Welt, ist mit ihm einer von den Grossen dahingegangen, dessen Name in der Ophthalmologie un-vergessen bleiben wird.

Dr. Hausch ild-Chcmnitz.

Offene Korrespondenz.

Epwidepung· auf Heppn Prof. Stillingrs Aufsatz: „Die Obliquus-fupehe; ein Wopt an meinen neuesten Geg-nep."

Von Dr. C. Hamburger, Schularzt in Berlin. (Eingereicht am 6. Dezember 1904.)

In No. 45 der Deutschen medizinischen Wochenschrift 1904 antvvortet Stilling auf meine Arbeit, betreffend die Grundlagen seiner ïheorie1) - oder richtiger gesagt, nieht auf die Arbeit selbst, sondern auf das Refernt eines Vortrages, den ich in der Berliner ophthalm. Gesellschaft im Mai gehalten hatte. Da der Leserkreis der D. m. W. - nach gefl. Mitteilung des Herausgebers - für die vorliegende Spezialfrage pur geringes Interesse hegt, so koonte mir zur Erwide Raum gewährt werden, um auszusprechen, dass ich meine Einwände $\operatorname{rrot}^{7} / 8$ Stillings Ironie nach wie vor aufrecht erhalte und in derjenigen Zeitschrift antworten würde, in der meine Arbeit publi-ziert sei.

S. Stillings diesmalige Antwort ist die zweite; die erste (Klin. Monatsbl.

') Diese Zeitschrift. 1904. Seite $351 \mathrm{ff}$. 